\title{
Well-posedness of the solution of the fractional semilinear pseudo-parabolic equation
}

\author{
Jiazhuo Cheng ${ }^{1}$ and Shaomei Fang ${ }^{1,2^{*}}$
}

\section{"Correspondence:}

dz90@scau.edu.cn

'Department of Applied

Mathematics, South China

Agricultural University, 510642,

Guangzhou, China

${ }^{2}$ Department of Applied

Mathematics, Yangtze Normal

University, 408100, Chongqing,

China

\begin{abstract}
This article concerns the Cauchy problem for the fractional semilinear pseudo-parabolic equation. Through the Green's function method, we prove the pointwise convergence rate of the solution. Furthermore, using this precise pointwise structure, we introduce a Sobolev space condition with negative index on the initial data and give the nonlinear critical index for blowing up.
\end{abstract}

Keywords: Fractional semilinear pseudo-parabolic equation; Green's function method; Pointwise; Negative index

\section{Introduction}

We consider the following Cauchy problem for the fractional semilinear pseudo-parabolic equation:

$$
\left\{\begin{array}{l}
u_{t}+k(-\triangle)^{a} u_{t}+(-\triangle)^{a} u=u^{p}, \quad x \in R^{n}, t>0 \\
\left.u\right|_{t=0}=u_{0}
\end{array}\right.
$$

where $p>0, k>0, u_{0}(x)$ is sufficiently smooth and nonnegative. If $k=0,(1.1)$ is the classical heat equation, see [1]. If $k>0,(1.1)$ is a pseudo-parabolic equation, see [2].

The pseudo-parabolic equation is used in diverse fields such as seepage theory of homogeneous liquid through cracked rock [3] (the coefficient of the third-order term represents the degree of cracks in the rock, and its decrease corresponds to the increase in the degree of cracking), the unidirectional propagation of nonlinear dispersive long waves $[4,5]$ (where $u$ is amplitude or curl), and the description of racial migration [6] (where $u$ is the population density). Because of the wide range of applications of pseudo-parabolic equations, they attract great attention of mathematicians.

Ting, Showalter, and Gopala Rao proved the existence and uniqueness of the solution on the initial boundary value problem and the Cauchy problem of linear pseudo-parabolic equations, see $[2,7,8]$. Since then, many scholars have paid great attention to the study of nonlinear pseudo-parabolic equations, including about existence, asymptotic behavior, decay of regularity and solutions, etc., see [9-12]. Recently, Yang Cao et al. proved

(c) The Author(s) 2020. This article is licensed under a Creative Commons Attribution 4.0 International License, which permits use, sharing, adaptation, distribution and reproduction in any medium or format, as long as you give appropriate credit to the original author(s) and the source, provide a link to the Creative Commons licence, and indicate if changes were made. The images or other third party material in this article are included in the article's Creative Commons licence, unless indicated otherwise in a credit line to the material. If material is not included in the article's Creative Commons licence and your intended use is not permitted by statutory regulation or exceeds the permitted use, you will need to obtain permission directly from the copyright holder. To view a copy of this licence, visit http://creativecommons.org/licenses/by/4.0/. 
the existence and blowing up of the solution of equation (1.1) at $a=1$, but the pointwise estimation of the solution was not discussed, see [13]. Later, Wang Weike et al. used the Green's function to improve [13] and $p$ only needs to satisfy $p>1+\frac{2}{n+s}$ instead of $p>1+\frac{2}{n}$. More specifically, they proved the pointwise estimation of the solution of equation (1.1) at $a=1$, also obtained the nonlinear critical index of the blowing up at $p>1+\frac{2}{n+s}$ by limiting the initial condition, see [14].

In the above research, they focused on integer order equations. The fractional dissipation operator $(-\triangle)^{a}$ can be regarded as the infinitesimal generators of Levy stable diffusion process. Compared with the integral differential equation, it can describe some physical phenomena more accurately, see [15-17]. Therefore, more and more scientists are devoted to the research of fractional differential equations, see [16-18].

Motivated by the above works, we study the pointwise estimate and exponential decay of the solution for problem (1.1) in the fractional order case. At present, there is little research on the pointwise estimate and exponential decay of the solution of this fractional equation, and the main difficulty stems from its fractional dissipation operator term. The structure of this article is organized as follows: In Sect. 2, we recall some preliminary results and show the main results of this paper. In Sect. 3, by Green's function method, we use the Green's function to express the solution of fractional equation (1.1) and get the pointwise estimate result of the Green's function. In Sect. 4, we obtain the pointwise estimate of fractional equation (1.1) with appropriate conditions $p, u_{0}$. In Sect. 5, we prove that the exponential decay of equation (1.1) still exists without $a=1$.

\section{Preliminaries and main results}

Let $C$ represent a generic positive constant, which may change from line to line. The norm of $L^{p}(\Omega)$ is written as $\|\cdot\|_{L^{p}(\Omega)}(1 \leq p \leq \infty)$. The notation $X$ is a Banach space with a norm $\|\cdot\|_{X} \cdot$

Definition 2.1 Suppose $f(x, t) \in L^{1}\left(R^{n}\right)$. Then the Fourier transform is as follows:

$$
\widehat{f}(\xi, t)=\int_{R^{n}} f(x, t) e^{-\sqrt{-1} x \cdot \xi} \mathrm{d} x
$$

its inverse Fourier transform is

$$
\left(\mathcal{F}^{-1} \widehat{f}\right)(x, t)=(2 \pi)^{-n} \int_{R^{n}} \widehat{f}(\xi, t) e^{\sqrt{-1} x \cdot \xi} \mathrm{d} \xi
$$

According to [19], we have the following two lemmas.

Lemma 2.1 If $\widehat{f}(\xi, t)$ has a compact support for $\xi$ satisfying

$$
\left|D_{\xi}^{\beta}\left(\xi^{\alpha} \widehat{f}(\xi, t)\right)\right| \leq C\left(|\xi|^{(|\alpha|+k-|\beta|)_{+}}+|\xi|^{(|\alpha|+k)} t^{\frac{|\beta|}{2}}\right)\left(1+t|\xi|^{2}\right)^{m} e^{-b|\xi|^{2} t}
$$

where $b>0, \alpha, \beta$ are any multi-indexes and $|\beta| \leq 2 N$, then

$$
\left|D_{x}^{\alpha} f(x, t)\right| \leq C_{N} t^{-n+|\alpha|+\frac{k}{2}} B_{N}(|x|, t),
$$


where $k$ and $m$ are any positive integers, $(a)_{+}=\max (0, a)$, and

$$
B_{N}(|x|, t)=\left(1+\frac{|x|^{2}}{1+t}\right)^{-N}
$$

Lemma 2.2 Let $\operatorname{supp} f(\xi) \subset O_{R}=:\{\xi,|\xi|>R\}$ and

$$
\left|D_{\xi}^{\beta} \widehat{f}(\xi)\right| \leq C|\xi|^{-1-|\beta|}
$$

then there exist distributions $f_{1}(x)$ and $f_{2}(x)$ satisfying

$$
f(x)=f_{1}(x)+f_{2}(x)+C_{0} \delta(x)
$$

where $C_{0}$ is a constant and $\delta(x)$ is the Dirac function. Furthermore, choosing $\varepsilon_{0}$ small enough, we have the estimate

$$
\begin{aligned}
& \left|D_{x}^{\alpha} f_{1}(x)\right| \leq C\left(1+|x|^{2}\right)^{-N}, \\
& \left\|f_{2}\right\|_{L_{1}} \leq C, \quad \operatorname{supp} f_{2}(x) \subset\left\{x ;|x|<2 \varepsilon_{0}\right\}
\end{aligned}
$$

for positive integer $2 N>n+|\alpha|$.

We make the following assumptions:

(H1) $u_{0} \in C^{\alpha+2}\left(R^{n}\right)$ for sufficiently small $u_{0}>0$;

(H2) $u_{0} \in W^{-s, 2}\left(R^{n}\right) \cap W^{-s, \infty}\left(R^{n}\right) \cap L^{\infty}\left(R^{n}\right) \cap L^{2}\left(R^{n}\right), 0 \leq s<n$, for sufficiently small $u_{0}>0$.

Based on the above assumptions, we draw the following conclusions.

Theorem 1 Let $p>p_{c}=1+\frac{2 a}{n}$, (H1) be satisfied. Then Cauchy problem (1.1) has the pointwise estimate of the solution $u$, satisfying

$$
\begin{aligned}
& |u| \leq 2 C(1+t)^{-\frac{n}{2 a}}\left(1+\frac{|x|^{2 a}}{1+t}\right)^{-N}, \\
& \left|D_{x}^{\alpha} u\right| \leq 2 C(1+t)^{-\frac{n}{2 a}-\frac{|\alpha|}{2 a}}\left(1+\frac{|x|^{2 a}}{1+t}\right)^{-N},
\end{aligned}
$$

where $N>0$ is an arbitrary constant, $C$ depends on the initial value $u_{0}$ and the parameter $p$.

Theorem 2 Suppose $p>p_{s}=1+\frac{2 a}{n+s},(H 2)$ hold. Then problem (1.1) has solution $u$ satisfying

$$
\|u\|_{L^{q\left(R^{n}\right)}} \leq C(1+t)^{-\frac{n}{2 a}\left(1-\frac{1}{q}\right)-\frac{s}{2 a}}, \quad q \in[2,+\infty)
$$

where $C$ depends on the initial value data and $p$. 


\section{Pointwise estimate of the Green's function}

In this section, we will consider the pointwise estimation of the solution to linear form of problem (1.1). We study the Green's function of Cauchy problem (1.1) and obtain the following:

$$
\left\{\begin{array}{l}
\partial_{t} G+k(-\triangle)^{a} G_{t}+(-\triangle)^{a} G=0, \quad x \in R^{n}, t>0, \\
\left.G\right|_{t=0}=\delta(x),
\end{array}\right.
$$

where $\delta(x)=\delta\left(x_{1}\right) \otimes \delta\left(x_{2}\right) \otimes \cdots \otimes \delta\left(x_{n}\right)$ is the Dirac function and $\otimes$ represents the tensor product. Considering the Fourier transform of equation (3.1) with respect to $x$, we get

$$
\left\{\begin{array}{l}
\partial_{t} \widehat{G}+k|\xi|^{2 a} \widehat{G}_{t}+|\xi|^{2 a} \widehat{G}=0, \quad \xi \in R^{n} \\
\left.\widehat{G}\right|_{t=0}=1
\end{array}\right.
$$

By solving the above equation directly, we know that

$$
\widehat{G}(\xi, t)=e^{\mu(\xi) t},
$$

where $\mu(\xi)=-\frac{|\xi|^{2 a}}{1+k|\xi|^{2 a}}$. Now we use frequency decomposition to obtain an estimate of the Green's function $G$. Let

$$
\begin{aligned}
& \chi_{1}(\xi)=\left\{\begin{array}{ll}
1, & |\xi| \leq \varepsilon, \\
0, & |\xi|>2 \varepsilon,
\end{array} \quad \in C^{\infty},\right. \\
& \chi_{3}(\xi)=\left\{\begin{array}{ll}
1, & |\xi| \geq R, \\
0, & |\xi|<R-1,
\end{array} \in C^{\infty},\right. \\
& \chi_{2}(\xi)=1-\chi_{1}(\xi)-\chi_{3}(\xi), \quad \in C^{\infty},
\end{aligned}
$$

where $\chi_{1}(\xi)$ and $\chi_{3}(\xi)$ are the smooth cut-off functions, $\varepsilon, R$ are any positive constants satisfying $2 \varepsilon<R-1$. Define $\widehat{G}_{i}(t, \xi)=\chi_{i} \widehat{G}(t, \xi), i=1,2,3$. From the literature [20], we know that the attenuation of the solution of the linear problem is mainly related to the low frequency part of $\widehat{G}(t, \xi)$. We use cut-off functions to divide the solution into three parts: low frequency, intermediate frequency, and high frequency.

Proposition 3.1 Let $\varepsilon$ be a sufficiently small constant. Then there exists a constant $C>0$ satisfying

$$
\left|D_{x}^{\alpha} G_{1}(x, t)\right| \leq C_{N}(1+t)^{-\frac{n+|\alpha|}{2 a}} B_{N}\left(|x|^{a}, t\right) .
$$

Proof In the case of low frequency, let $0<|\xi|<2 R$, then $\widehat{G}$ has compact support. Taking into account (3.3) and Lemma 2.1, there is

$$
\left|D_{\xi}^{\beta} \xi^{\alpha} \chi_{1}(\xi) \widehat{G}(\xi, t)\right| \leq C\left(|\xi|^{(|\alpha|-|\beta|)_{+}}+|\xi|^{|\alpha|} t^{\mid \frac{|\beta|}{2 a}}\right)\left(1+t|\xi|^{2 a}\right)^{\beta} e^{-b|\xi|^{2 a} t}
$$

for $\forall|\beta| \leq 2 N$. Then is (3.7) established. 
Actually, we can discuss obtaining (3.7) in two cases.

(1) If $|\beta| \leq|\alpha|$, we find

$$
\begin{aligned}
\left|x^{\beta} D_{x}^{\alpha} \chi_{1}(D) G\right| & =C\left|\int e^{i x \xi} D_{\xi}^{\beta}\left(\xi^{\alpha} \chi_{1}(\xi) \widehat{G}(\xi, t)\right) \mathrm{d} \xi\right| \\
& \leq C \int\left(|\xi|^{(|\alpha|-|\beta|)_{+}}+|\xi|^{|\alpha|} t^{\frac{|\beta|}{2 a}}\right)\left(1+t|\xi|^{2 a}\right)^{\beta}\left|e^{i x \xi}\right| e^{-b|\xi|^{2 a} t} \mathrm{~d} \xi \\
& \leq C \int|\xi|^{|\alpha|}\left(|\xi|^{-|\beta|_{+}}+t^{\frac{|\beta|}{2 a}}\right)\left(1+t|\xi|^{2 a}\right)^{\beta} e^{-b|\xi|^{2 a} t} \mathrm{~d} \xi \\
& \leq C t^{-\frac{n+|\alpha|-|\beta|}{2 a}} .
\end{aligned}
$$

(2) If $|\beta|>|\alpha|$, we obtain

$$
\begin{aligned}
\left|x^{\beta} D_{x}^{\alpha} \chi_{1}(D) G\right| & \leq C\left(1+t^{-\frac{|\alpha|-|\beta|}{2 a}}\right) t^{-\frac{n}{2 a}} \\
& \leq C(1+t)^{-\frac{|\alpha|-|\beta|}{2 a}} t^{-\frac{n}{2 a}} \\
& \leq C(1+t)^{\frac{|\beta|}{2 a}} t^{-\frac{n+|\alpha|}{2 a}}
\end{aligned}
$$

On the other hand, if $|x|^{2 a} \leq 1+t$, let $|\beta|=0$, we have

$$
\left|D_{x}^{\alpha} \chi_{1}(D) G\right| \leq C t^{-\frac{n+|\alpha|}{2 a}} .
$$

If $|x|^{2 a}>1+t$, let $|\beta|=2 a N$, we see that

$$
\left|D_{x}^{\alpha} \chi_{1}(D) G\right| \leq C t^{-\frac{n+|\alpha|}{2 a}} \min \left(1, \frac{(1+t)^{N}}{|x|^{2 a N}}\right)
$$

Because

$$
1+\frac{|x|^{2 a}}{1+t} \leq \begin{cases}2, & |x|^{2 a} \leq 1+t \\ 2 \frac{|x|^{2 a}}{1+t}, & |x|^{2 a}>1+t\end{cases}
$$

we know that

$$
\min \left(1, \frac{(1+t)^{N}}{|x|^{2 a N}}\right) \leq 2^{N} B_{N}\left(|x|^{a}, t\right) .
$$

Then, with the help of (3.11)-(3.12) and (3.14), we infer (3.7).

Next, we estimate $G_{2}(x, t)$.

Proposition 3.2 Suppose that $\varepsilon$ and $R$ are fixed constants. Then

$$
\left|D_{x}^{\alpha} G_{2}(x, t)\right| \leq C e^{-\frac{t}{2 m_{0}}} B_{N}\left(|x|^{a}, t\right),
$$

where $m_{0}$ is a positive constant. 
Proof Choosing $m$ sufficiently large and $m>\frac{1}{2}\left(\frac{1}{|\varepsilon|^{2 a}}+k\right)$. If $\varepsilon \leq|\xi| \leq R$, it is easy to see that $\mu(\xi) \leq-\frac{1}{2 m}$. This analysis reflects that

$$
\left|\widehat{G_{2}}\right|=\left|\chi_{2}(\xi) \widehat{G}\right| \leq C e^{-\frac{t}{2 m}}
$$

From (3.16), there holds

$$
\begin{aligned}
\left|D_{x}^{\alpha} G_{2}(x, t)\right| & \leq C\left|\int_{\varepsilon \leq \xi \leq R} e^{i x \xi}\left(\xi^{\alpha} \widehat{G_{2}}(\xi, t)\right) \mathrm{d} \xi\right| \\
& \leq C e^{-\frac{t}{2 m}} \int_{\varepsilon \leq \xi \leq R}|\xi|^{\alpha} \mathrm{d} \xi \\
& \leq C e^{-\frac{t}{2 m}}
\end{aligned}
$$

Now, we apply mathematical induction to prove the following inequality:

$$
\left|D_{\xi}^{\beta} \widehat{G_{2}}(\xi, t)\right| \leq C(1+t)^{|\beta|} e^{-\frac{t}{2 m}}
$$

Obviously, the above formula holds when $|\beta|=0$. Suppose that $|\beta| \leq l-1$, the above formula still holds. Then we will prove that the formula of (3.18) also holds for $|\beta| \leq l$. Taking the Fourier transform of (3.1) with respect to $x$ and multiplying it with $\chi_{2}(\xi)$, we get

$$
\left\{\begin{array}{l}
\partial_{t} \widehat{G_{2}}(\xi, t)-\mu(\xi) \widehat{G_{2}}(\xi, t)=0 \\
\widehat{G_{2}}(\xi, 0)=\chi_{2}(\xi)
\end{array}\right.
$$

Using $D_{\xi}^{\beta}$ to equation (3.19), we can obtain that

$$
\left\{\begin{array}{l}
\partial_{t} D_{\xi}^{\beta} \widehat{G_{2}}(\xi, t)-\mu(\xi) D_{\xi}^{\beta} \widehat{G_{2}}(\xi, t)=F(\xi, t), \\
\widehat{G}(\xi, 0)=a_{0}
\end{array}\right.
$$

where $F(\xi, t)=\sum_{\beta_{1}+\beta_{2}=\beta,\left|\beta_{1}\right| \neq 0} \frac{\beta !}{\beta_{1} ! \beta_{2} !}\left(D_{\xi}^{\beta_{1}} \mu(\xi) D_{\xi}^{\beta_{2}} \widehat{G_{2}}(\xi, t)\right)$ and $a_{0}$ is a polynomial of $|\xi|$. Considering $|\beta|=l$, we see that

$$
D_{\xi}^{\beta} \widehat{G_{2}}(\xi, t)=a_{0} \widehat{G_{2}}(\xi, t)+\int_{0}^{t} \widehat{G_{2}}(\xi, t-s) F(\xi) \mathrm{d} s .
$$

By induction we have

$$
\begin{aligned}
\left|D_{\xi}^{\beta} \widehat{G_{2}}(\xi, t)\right| & \leq C e^{-\frac{t}{2 m}}+C \int_{0}^{t} e^{-\frac{t-s}{2 m}}(1+t)^{|\beta|-1} e^{-\frac{s}{2 m}} \mathrm{~d} s \\
& \leq C(1+t)^{|\beta|} e^{-\frac{t}{2 m}}
\end{aligned}
$$


Therefore, for each $1 \leq|\beta| \leq l$, we find

$$
\begin{aligned}
\left|x^{\beta} D_{x}^{\alpha} G_{2}(x, t)\right| & \leq C\left|\int_{R^{n}} e^{i x \xi} D_{\xi}^{\beta}\left(\xi^{\alpha} \widehat{G_{2}}(\xi, t)\right) \mathrm{d} s\right| \\
& \leq C e^{-\frac{t}{2 m}}(1+t)^{|\beta|} \int_{\varepsilon \leq|\beta| \leq R}|\xi|^{|\alpha|}+|\xi|^{|| \alpha|-| \beta||} \mathrm{d} \xi \\
& \leq C e^{-\frac{t}{2 m}}(1+t)^{|\beta|} \\
& \leq C e^{-\frac{t}{2 m_{0}}}(1+t)^{\frac{|\beta|}{2 a}}
\end{aligned}
$$

where $m_{0} \in(0, m)$. Taking into account (3.17) and (3.23) (let $\left.|\beta|=2 N\right)$, we deduce

$$
\left|D_{x}^{\alpha} G_{2}(x, t)\right| \leq C e^{-\frac{t}{2 m_{0}}} \min \left(1, \frac{(1+t)^{2 N}}{|x|^{2 N}}\right) .
$$

Considering (3.14) and (3.24), we obtain the desired result.

Now considering the high frequency part $G_{3}(x, t)$.

Proposition 3.3 Let $R$ be a sufficiently small constant. Then

$$
\left|D_{x}^{\alpha}\left(G_{3}(x, t)-F_{l}\right)\right| \leq C(1+t)^{-\frac{n+|\alpha|}{2 a}} B_{N}\left(|x|^{a}, t\right),
$$

where $b$ is a positive constant and

$$
F_{l}=\chi_{3}(D)\left[e^{-\frac{t}{k}}\left(\delta(x)+\sum_{j=1}^{l} p_{j}(t)(-\triangle)^{-a j}\right)\right], \quad l=\frac{|\alpha|+n}{2 a}
$$

is the distribution.

Proof Denote

$$
\rho=\frac{1}{|\xi|^{2 a}}, \quad h(\rho)=e^{-\frac{t}{|\rho|+k}}
$$

Expanded in Taylor's series at $|\rho| \rightarrow 0$, we infer

$$
h(\rho)=e^{-\frac{t}{k}}\left(1+\frac{t \rho}{k^{2}}+\frac{t^{2} \rho^{2}}{2 k^{4}}-\frac{t \rho^{2}}{2 k^{3}}+\cdots\right) .
$$

Then

$$
e^{-\frac{t|\xi|^{2 a}}{1+k|\xi|^{2 a}}}=e^{-\frac{t}{k}}\left(1+\sum_{j=1}^{l} p_{j}(t)\left(|\xi|^{2 a}\right)^{-j}\right) \chi_{3}(\xi)+R(\xi, t)
$$

where $p_{j}(t)$ is a polynomial of degree $j$.

Let

$$
F_{l}=\chi_{3}(D)\left[e^{-\frac{t}{k}}\left(\delta(x)+\sum_{j=1}^{l} p_{j}(t)(-\Delta)^{-a j}\right)\right], \quad l=\frac{|\alpha|+n}{2 a}
$$


With the help of Lemma 2.2 and choosing $R$ big enough, it is easy to see that

$$
\left|D_{x}^{\alpha}\left(G_{3}(x, t)-F_{l}\right)\right| \leq C(1+t)^{-\frac{n+|\alpha|}{2 a}} B_{N}\left(|x|^{a}, t\right) .
$$

In conclusion, we use the following lemma to explain the estimate of the regular part of $G$.

Lemma 3.1 Let $G$ be the solution of the linear form of Cauchy problem (1.1). Then

$$
\left|D_{x}^{\alpha}\left(G(x, t)-F_{l}\right)\right| \leq C(1+t)^{-\frac{n+|\alpha|}{2 a}} B_{N}\left(|x|^{a}, t\right)
$$

where $F_{l}$ is the distribution and

$$
F_{l}=\chi_{3}(D)\left[e^{-\frac{t}{k}}\left(\delta(x)+\sum_{j=1}^{l} p_{j}(t)(-\triangle)^{-a j}\right)\right], \quad l=\frac{|\alpha|+n}{2 a}
$$

Proof Note that

$$
\begin{aligned}
\left|D_{x}^{\alpha}\left(G(x, t)-F_{l}\right)(x, t)\right| \leq & \left|D_{x}^{\alpha} G_{1}(x, t)\right|+\left|D_{x}^{\alpha} G_{2}(x, t)\right| \\
& +\left|D_{x}^{\alpha}\left(G_{3}(x, t)-F_{l}\right)(x, t)\right| .
\end{aligned}
$$

Considering Proposition 3.1, Proposition 3.2, Proposition 3.3, and (3.34), we have (3.32). Then Lemma 3.1 is proved.

Lemma 3.2 Assume that $t>0, m>\frac{n}{2 a}$, then

$$
\int_{R^{n}}\left(1+\frac{|x|^{2 a}}{1+t}\right)^{-m} \mathrm{~d} x \leq C(1+t)^{\frac{n}{2 a}}
$$

Proof Let $w=|x|$, we infer

$$
\begin{aligned}
\int_{R^{n}}\left(1+\frac{|x|^{2 a}}{1+t}\right)^{-m} \mathrm{~d} x & \leq C \int_{0}^{\infty}\left(1+\frac{w^{2 a}}{1+t}\right)^{-m} w^{n-1} \mathrm{~d} w \\
& \leq C(1+t)^{\frac{n}{2 a}} \int_{0}^{\infty}\left(1+\frac{w}{(1+t)^{\frac{1}{2 a}}}\right)^{-2 a m+n-1} \mathrm{~d} \frac{w}{(1+t)^{\frac{1}{2 a}}} \\
& \leq C(1+t)^{\frac{n}{2 a}}
\end{aligned}
$$

Using Hausdorff-Young's inequality, the following lemma of Green's function is easily obtained.

Lemma 3.3 If $p \in[1, \infty]$, then

$$
\left\|D_{x}^{\alpha}\left(G-F_{l}\right)(t, \cdot)\right\|_{L^{p}\left(R^{n}\right)} \leq C(1+t)^{-\frac{n}{2 a}\left(1-\frac{1}{p}\right)-\frac{|\alpha|}{2 a}}
$$

for any multi-indexes $\alpha$. 
Proof If $p \in[1, \infty)$, it follows from (3.25) that

$$
\begin{aligned}
\left\|D_{x}^{\alpha}\left(G-F_{l}\right)(t, \cdot)\right\|_{L^{p}\left(R^{n}\right)} & =\left.\left.\left|\int_{R^{n}}\right| D_{x}^{\alpha}\left(G-F_{l}\right)(t, \cdot)\right|^{p} \mathrm{~d} x\right|^{\frac{1}{p}} \\
& \leq C(1+t)^{-\frac{n+|\alpha|}{2 a}}\left(\int_{R^{n}}\left|B_{N}\left(|x|^{a}, t\right)\right|^{p} \mathrm{~d} x\right)^{\frac{1}{p}} \\
& \leq C(1+t)^{-\frac{n}{2 a}\left(1-\frac{1}{p}\right)-\frac{|\alpha|}{2 a}} .
\end{aligned}
$$

If $p=\infty$, by (3.25), we conclude that

$$
\begin{aligned}
\left\|D_{x}^{\alpha}\left(G-F_{l}\right)(t, \cdot)\right\|_{L^{p}\left(R^{n}\right)} & =\operatorname{ess} \sup \left|D_{x}^{\alpha}\left(G-F_{l}\right)(t, \cdot)\right| \\
& \leq \operatorname{ess} \sup \left\{C(1+t)^{-\frac{n+|\alpha|}{2 a}} B_{N}\left(|x|^{a}, t\right)\right\} \\
& \leq C(1+t)^{-\frac{n}{2 a}\left(1-\frac{1}{p}\right)-\frac{|\alpha|}{2 a}}
\end{aligned}
$$

\section{Pointwise estimation of the solution}

In this section, we get the pointwise estimate of the solution under appropriate conditions of $u_{0}, p$.

Lemma 4.1 Assume that $|y| \leq M, t \geq 4 M^{2}, N>0$, then

$$
\left(1+\frac{|y-x|^{2 a}}{1+t}\right)^{-N} \leq C_{N}\left(1+\frac{|x|^{2 a}}{1+t}\right)^{-N} .
$$

Proof (1) If $|x|^{2 a} \leq 1+t$, then

$$
\frac{|x|^{2 a}}{1+t} \leq 1
$$

and

$$
\left(1+\frac{|x|^{2 a}}{1+t}\right)^{-N} \geq 2^{-N} .
$$

On the other hand,

$$
\left(1+\frac{|x-y|^{2 a}}{1+t}\right)^{-N} \leq 1
$$

It follows from (4.3) and (4.4) that

$$
\left(1+\frac{|x|^{2 a}}{1+t}\right)^{-N} \leq 2^{N}\left(1+\frac{|x|^{2 a}}{1+t}\right)^{-N} .
$$

(2) If $|x|^{2 a}>1+t$, then

$$
|x-y| \geq|x|-|y| \geq \sqrt{1-t}-|y| \geq 0
$$


and

$$
\begin{aligned}
|x-y|^{2 a} & \geq(|x|-|y|)^{2 a}=\left(|x|^{2}-2|x||y|+|y|^{2}\right)^{a} \\
& =\left(\frac{|x|^{2}}{2}+\frac{(|x|-2|y|)^{2}}{2}-|y|^{2}\right)^{a} \geq\left(\frac{|x|^{2}}{4}+\left(\frac{t}{4}-M^{2}\right)\right)^{a} .
\end{aligned}
$$

From $\frac{t}{4}-M^{2} \geq 0$, the proof is obtained.

Proof of Theorem 1 With the help of Lemma 3.3 and considering (1.1), from Green's function, we have

$$
\begin{aligned}
u & =\widehat{T} u=G(t) * u_{0}+\int_{0}^{t} H(t-s, \cdot) * F(u(s, \cdot)) \mathrm{d} s \\
& =G(t) * u_{0}+\int_{0}^{t} \int_{R^{n}} H(x-y, t-s) * F(u(y, s)) \mathrm{d} y \mathrm{~d} s
\end{aligned}
$$

where the symbol $*$ represents convolution, $F(u)=u^{p}$, and $H$ satisfies

$$
\widehat{H}=\frac{1}{1+k|\xi|^{2 a}} \widehat{G} .
$$

Applying the inverse Fourier transform, we deduce

$$
H=K_{k} * G, \quad K_{k}(x)=(4 \pi)^{-\frac{n}{2 a}} \int_{0}^{\infty} e^{-\gamma_{1} s-\frac{|x|^{2 a}}{4 s}} s^{-\frac{n}{2 a}} \mathrm{~d} s
$$

Obviously, the estimated value on $\widehat{G}$ is also correct on $\widehat{H}$.

By using $D_{x}^{\alpha}$ to equation (4.8), we get

$$
\begin{aligned}
D_{x}^{\alpha} u(x, t) & =D_{x}^{\alpha} G * u_{0}+\int_{0}^{t} \int_{R^{n}} H(x-y, t-s) D_{x}^{\alpha} F(u(y, s)) \mathrm{d} y \mathrm{~d} s \\
& =D_{x}^{\alpha} G * u_{0}+\int_{0}^{t} \int_{R^{n}} D_{x}^{\alpha} H(x-y, t-s) F(u(y, s)) \mathrm{d} y \mathrm{~d} s .
\end{aligned}
$$

Define

$$
\begin{aligned}
& \phi=(1+t)^{-\frac{n}{2 a}} B_{N}\left(|x|^{a}, t\right), \\
& \phi_{\alpha}=(1+t)^{-\frac{n+|\alpha|}{2 a}} B_{N}\left(|x|^{a}, t\right), \\
& M(t)=\sup _{0 \leq s \leq t, x \in R}|u(x, s)| \phi^{-1}(x, s) .
\end{aligned}
$$

Then

$$
\begin{aligned}
& u \leq M \cdot \phi \\
& u(x, t) \leq G * u_{0}+M^{p} \int_{0}^{t} \int_{R^{n}} H(x-y, t-s) \phi^{p}(y, s) \mathrm{d} y \mathrm{~d} s \\
& D_{x}^{\alpha} u(x, t) \leq D_{x}^{\alpha} G * u_{0}+M^{p} \int_{0}^{t} \int_{R^{n}} D_{x}^{\alpha} H(x-y, t-s) \phi^{p}(y, s) \mathrm{d} y \mathrm{~d} s .
\end{aligned}
$$


First of all, we discuss the singular part. By $(H 2)$, following [20], we have

$$
\begin{aligned}
\left|D_{x}^{\alpha} F_{l} * u_{0}\right| & \leq C \varepsilon e^{-\frac{t}{2 a k}}\left(1+|x|^{2 a}\right)^{-N} \\
& \leq C \varepsilon(1+t)^{-\frac{n+|\alpha|}{2 a}}\left(1+\frac{|x|^{2 a}}{1+t}\right)^{-N} \\
& \leq C \varepsilon \phi_{\alpha} .
\end{aligned}
$$

Secondly, we consider the nonsingular part. Since $\left|u_{0}\right| \leq\left(1+|y|^{2 a}\right)^{-N}$, supp $u_{0} \subset\{|y| \leq$ $M$ \}, according to the definition of tight support, we can know $u_{0}$ has a compact support. If $t$ is large enough, we find

$$
\begin{aligned}
\left|D_{x}^{\alpha}\left(G-F_{l}\right) * u_{0}\right| & \leq C(1+t)^{-\frac{n+|\alpha|}{2 a}} \int_{R^{n}}\left(1+\frac{|y-x|^{2 a}}{1+t}\right)^{-N}\left(1+\frac{|y|^{2 a}}{1+t}\right)^{-N} \mathrm{~d} y \\
& \leq C(1+t)^{-\frac{n+|\alpha|}{2 a}}\left(1+\frac{|x|^{2 a}}{1+t}\right)^{-N} \int_{R^{n}}\left(1+\frac{|y|^{2 a}}{1+t}\right)^{-N} \mathrm{~d} y \\
& \leq C \varepsilon \phi_{\alpha} .
\end{aligned}
$$

Then

$$
\left|D_{x}^{\alpha} G * u_{0}\right| \leq C \varepsilon \phi_{\alpha}
$$

Here we still divide the nonlinear term into a singular part and a nonsingular part. Define

$$
\begin{aligned}
\int_{0}^{t} \int_{R^{n}} D_{x}^{\alpha} H(x-y, t-s) \phi^{p}(y, s) \mathrm{d} y \mathrm{~d} s \leq & \int_{0}^{t} \int_{R^{n}} D_{x}^{\alpha}\left(G-F_{l}\right) \phi^{p}(y, s) \mathrm{d} y \mathrm{~d} s \\
& +\int_{0}^{t} \int_{R^{n}} D_{x}^{\alpha} F_{l} \phi^{p}(y, s) \mathrm{d} y \mathrm{~d} s \\
& =\psi_{1,1}+\psi_{1,2},
\end{aligned}
$$

where $\psi_{1,1}=\int_{0}^{t} \int_{R^{n}} D_{x}^{\alpha}\left(G-F_{l}\right) \phi^{p}(y, s) \mathrm{d} y \mathrm{~d} s, \psi_{1,2}=\int_{0}^{t} \int_{R^{n}} D_{x}^{\alpha} F_{l} \phi^{p}(y, s) \mathrm{d} y \mathrm{~d} s$.

Estimating the nonsingular part. Recalling Lemma 3.1, it shows that

$$
\begin{aligned}
\psi_{1,1} \leq & C \int_{0}^{t} \int_{R^{n}}(1+t-s)^{-\frac{n+|\alpha|}{2 a}}\left(1+\frac{|x-y|^{2 a}}{1+t-s}\right)^{-N} \\
& \times(1+s)^{-\frac{n p}{2 a}}\left(1+\frac{|y|^{2 a}}{1+s}\right)^{-N p} \mathrm{~d} y \mathrm{~d} s .
\end{aligned}
$$

Denote $\Omega=[0, t] \times R^{n}, \Omega^{1}=\Omega \cap\left\{s \geq \frac{t}{2}\right\}, \Omega^{2}=\Omega \cap\left\{s \leq \frac{t}{2}\right\}$. We will discuss it in two cases:

(1) If $|x|^{2 a} \leq 1+t$, then

$$
\begin{aligned}
\psi_{1,1} \leq & C \int_{\Omega^{1}}(1+t-\tau)^{-\frac{n+|\alpha|}{2 a}}\left(1+\frac{|x-y|^{2 a}}{1+t-\tau}\right)^{-N}(1+\tau)^{-\frac{n p}{2 a}} \\
& \times\left(1+\frac{|y|^{2 a}}{1+\tau}\right)^{-N p} \mathrm{~d} y \mathrm{~d} \tau+C \int_{\Omega^{2}}(1+t-\tau)^{-\frac{n}{2 a}}\left(1+\frac{|x-y|^{2 a}}{1+t-\tau}\right)^{-N}
\end{aligned}
$$




$$
\begin{aligned}
& \times(1+\tau)^{-\frac{n p+|\alpha|}{2 a}}\left(1+\frac{|y|^{2 a}}{1+\tau}\right)^{-N p} \mathrm{~d} y \mathrm{~d} \tau \\
\leq & C \int_{\frac{t}{2}}^{t}(1+t-\tau)^{-\frac{n+|\alpha|}{2 a}}(1+\tau)^{-\frac{n p}{2 a}}(1+\tau)^{\frac{n}{2 a}} \mathrm{~d} \tau \\
& +C \int_{0}^{\frac{t}{2}}(1+t-\tau)^{-\frac{n}{2 a}}(1+\tau)^{-\frac{n p+|\alpha|}{2 a}}(1+\tau)^{\frac{n}{2 a}} \mathrm{~d} \tau \\
\leq & C(1+t)^{-\frac{n+n p-n}{2 a}+1-\frac{|\alpha|}{2 a}} \\
\leq & C(1+t)^{-\frac{n p}{2 a}+1-\frac{|\alpha|}{2 a}}\left(1+\frac{|x|^{2 a}}{1+t}\right)^{-n} .
\end{aligned}
$$

(2) If $|x|^{2 a}>1+t$, then

$$
\begin{aligned}
\psi_{1,1} \leq & C \int_{\Omega^{1}}(1+t-\tau)^{-\frac{n+|\alpha|}{2 a}}\left(1+\frac{|x-y|^{2 a}}{1+t-\tau}\right)^{-N}(1+\tau)^{-\frac{n p}{2 a}} \\
& \times\left(1+\frac{|y|^{2 a}}{1+\tau}\right)^{-N p} \mathrm{~d} y \mathrm{~d} \tau+C \int_{\Omega^{2}}(1+t-\tau)^{-\frac{n}{2 a}}\left(1+\frac{|x-y|^{2 a}}{1+t-\tau}\right)^{-N} \\
& \times(1+\tau)^{-\frac{n p+|\alpha|}{2 a}}\left(1+\frac{|y|^{2 a}}{1+\tau}\right)^{-N p} \mathrm{~d} y \mathrm{~d} \tau \\
\leq & C \int_{\Omega^{1}}(1+t-\tau)^{-\frac{n+|\alpha|}{2 a}}\left(1+\frac{|x|^{2 a}}{1+t-\tau}\right)^{-N}(1+\tau)^{-\frac{n p}{2 a}} \\
& \times\left(1+\frac{|y|^{2 a}}{1+\tau}\right)^{-N p} \mathrm{~d} y \mathrm{~d} \tau+C \int_{\Omega^{2}}(1+t-\tau)^{-\frac{n}{2 a}}\left(1+\frac{|x|^{2 a}}{1+t-\tau}\right)^{-N} \\
& \times(1+\tau)^{-\frac{n p+|\alpha|}{2 a}}\left(1+\frac{|x|^{2 a}}{1+\tau}\right)^{-N p} \mathrm{~d} y \mathrm{~d} \tau \\
\leq & C\left(1+\frac{|x|^{2 a}}{1+\tau}\right)^{-n} \int_{\frac{t}{2}}^{t}(1+t-\tau)^{-\frac{n+|\alpha|}{2 a}}(1+\tau)^{-\frac{n p}{2 a}}(1+\tau)^{\frac{n}{2 a}} \mathrm{~d} \tau \\
\leq & C(1+t)^{-\frac{n p}{2 a}+1-\frac{|\alpha|}{2 a}}\left(1+\frac{|x|^{2 a}}{1+t}\right)^{-n} \cdot \\
& +C\left(1+\frac{|x|^{2 a}}{1+\tau}\right)^{-n} \int_{0}^{\frac{t}{2}}(1+t-\tau)^{-\frac{n}{2 a}}(1+\tau)^{-\frac{n p+|\alpha|}{2 a}}(1+\tau)^{\frac{n}{2 a}} \mathrm{~d} \tau
\end{aligned}
$$

From (4.19)-(4.20), we can get

$$
\psi_{1,1} \leq C(1+t)^{-\frac{n p}{2 a}+1-\frac{|\alpha|}{2 a}} B_{N}\left(|x|^{a}, t\right)
$$

Due to $p>1+\frac{2 a}{n}$, we deduce

$$
(1+t)^{-\frac{n p}{2 a}+1-\frac{|\alpha|}{2 a}} \leq(1+t)^{-\frac{n}{2 a}-\frac{|\alpha|}{2 a}}
$$

Estimating the singular part. By [19], we know that

$$
\psi_{1,2}=\int_{0}^{t} \int_{R^{n}} D_{x}^{\alpha} F_{l} \phi^{p}(y, s) \mathrm{d} y \mathrm{~d} s
$$




$$
\begin{aligned}
& \leq \int_{0}^{t} e^{-\frac{t}{2 k a}} \phi_{\alpha}^{p} \mathrm{~d} s \\
& \leq C \phi_{\alpha} .
\end{aligned}
$$

Coming back to the whole solution, we find

$$
\begin{aligned}
|u(x, t)| & \leq\left|G * u_{0}\right|+\left|\int_{0}^{t} \int_{R^{n}} H(x-y, t-s) F(u(y, s)) \mathrm{d} y \mathrm{~d} s\right| \\
& \leq C\left(\varepsilon+M^{p}\right) \phi, \\
\left|D_{x}^{\alpha} u(x, t)\right| & \leq C\left(\varepsilon+M^{p}\right) \phi_{\alpha} .
\end{aligned}
$$

Therefore,

$$
M \leq C\left(\varepsilon+M^{p}\right) .
$$

Since $M(0) \leq C \varepsilon$, applying the continuity method, we get that

$$
M(t) \leq 2 C \varepsilon, \quad \forall t \in[0,+\infty)
$$

By the above inequality, we have

$$
\begin{aligned}
& \left|D_{x}^{\alpha} u\right| \leq M \phi_{\alpha} \leq 2 C \varepsilon \phi_{\alpha}, \\
& |u| \leq 2 C \varepsilon(1+t)^{-\frac{n}{2 a}}\left(1+\frac{|x|^{2 a}}{1+t}\right)^{-N}, \\
& \left|D_{x}^{\alpha} u\right| \leq 2 C \varepsilon(1+t)^{-\frac{n+|\alpha|}{2 a}}\left(1+\frac{|x|^{2 a}}{1+t}\right)^{-N} .
\end{aligned}
$$

\section{Improvement of the initial data}

In this section we consider the Cauchy problem of (1.1). It shows that the limit of the parameter $p$ can be weaker when the initial conditions become stronger. Since we have known the proof of the existence and uniqueness of the solution, we will not discuss it. Only for attenuation of the decay estimate.

Definition 5.1 Suppose that operator $\Lambda^{s}, s \in R^{n}$, satisfies

$$
\Lambda^{s} f(x)=(2 \pi)^{-n} \int_{R^{n}}|\xi| \widehat{f} \widehat{f}(\xi) e^{\sqrt{-1} x \cdot \xi} \mathrm{d} \xi
$$

and Sobolev space $W^{-s, p}$ indicates

$$
W^{-s, p}=\left\{f \mid\left\|\Lambda^{-s} f\right\|_{L^{p}\left(R^{n}\right)} \leq C\right\}
$$

Lemma 5.1 Let $0<s<n, 1<p<q<\infty, \frac{1}{q}+\frac{s}{n}=\frac{1}{p}$, then

$$
\left\|\Lambda^{-s} f\right\|_{L^{q}\left(R^{n}\right)} \leq C_{p, q}\|f\|_{L^{q}\left(R^{n}\right)},
$$

where $C_{p, q}$ is a constant depending on $p, q$. 
Through [21, p. 99, Theorem 1], Lemma 5.1 can be proved.

Proof of Theorem 2 We divide it into a singular part and a nonsingular part:

$$
u=G * u_{0}+\int_{0}^{t} H * u^{p} \mathrm{~d} \tau=u_{N}+u_{S}
$$

where

$$
\begin{aligned}
& u_{N}=\left(G-F_{l}\right) * u_{0}+\int_{0}^{t} \frac{G-F_{l}}{1+k(-\triangle)^{a}} * u^{p} \mathrm{~d} \tau, \\
& u_{S}=F_{l} * u_{0}+\int_{0}^{t} \frac{F_{l}}{1+k(-\triangle)^{a}} * u^{p} \mathrm{~d} \tau .
\end{aligned}
$$

Now, let us start from the nonsingular part, we obtain

$$
\begin{aligned}
u_{N} & =\left(G-F_{l}\right) * u_{0}+\int_{0}^{t} \frac{G-F_{l}}{1+k(-\triangle)^{a}} * u^{p} \mathrm{~d} \tau \\
& =\Lambda^{s}\left(G-F_{l}\right) * \Lambda^{-s} u_{0}+\int_{0}^{t} \Lambda^{s} \frac{G-F_{l}}{1+k(-\triangle)^{a}} * \Lambda^{-s} u^{p} \mathrm{~d} \tau
\end{aligned}
$$

Using Young's inequality, we have

$$
\begin{aligned}
\left\|u_{N}\right\|_{L^{q}\left(R^{n}\right)} \leq & \left\|\Lambda^{s}\left(G-F_{l}\right) * \Lambda^{-s} u_{0}\right\|_{L^{q}\left(R^{n}\right)} \\
& +\left\|\int_{0}^{t} \Lambda^{s}\left(G-F_{l}\right) * \Lambda^{-s} u^{p} \mathrm{~d} \tau\right\|_{L^{q}\left(R^{n}\right)} \\
\leq & \left\|\Lambda^{s}\left(G-F_{l}\right) * \Lambda^{-s} u_{0}\right\|_{L^{q}\left(R^{n}\right)} \\
& +\int_{0}^{t}\left\|\Lambda^{s}\left(G-F_{l}\right)\right\|_{L^{\frac{n q}{n+q}\left(R^{n}\right)}}\left\|\Lambda^{-s} u^{p}\right\|_{L^{\frac{n}{n-1}\left(R^{n}\right)}} \mathrm{d} \tau .
\end{aligned}
$$

Based on the initial condition and estimate of the linear part, we see that

$$
\left\|\Lambda^{s}\left(G-F_{l}\right)\right\|_{L^{q\left(R^{n}\right)}} \leq C(1+t)^{-\frac{n}{2 a}\left(1-\frac{1}{q}\right)-\frac{s}{2 a}}
$$

and

$$
\left\|\Lambda^{s}\left(G-F_{l}\right) * \Lambda^{-s} u_{0}\right\|_{L^{q\left(R^{n}\right)}} \leq C(1+t)^{-\frac{n}{2 a}\left(1-\frac{1}{q}\right)-\frac{s}{2 a}} .
$$

Recalling Lemma 5.1, there holds

$$
\left\|\Lambda^{-s} u^{p}\right\|_{L^{\frac{n}{n-1}\left(R^{n}\right)}} \leq C\|u\|_{L_{\frac{n p}{n+s-1}\left(R^{n}\right)}^{p}}^{p} \leq C\left[(1+t)^{-\frac{n}{2 a}\left(1-\frac{n+s-1}{n p}\right)-\frac{s}{2 a}}\right]^{p} .
$$


Taking into account (5.8) and (5.10), we get

$$
\begin{aligned}
& \int_{0}^{t}\left\|\Lambda^{s}\left(G-F_{l}\right)\right\|_{L^{\frac{n q}{n+q}\left(R^{n}\right)}}\left\|\Lambda^{-s} u^{p}\right\|_{L^{\frac{n}{n-1}\left(R^{n}\right)}} \mathrm{d} s \\
& \quad \leq C \int_{0}^{t}(1+t-s)^{-\frac{n}{2 a}\left(1-\frac{n+q}{n q}\right)-\frac{s}{2 a}}\left[(1+t)^{-\frac{n}{2 a}\left(1-\frac{n+s-1}{n p}\right)-\frac{s}{2 a}}\right]^{p} \mathrm{~d} s \\
& \quad \leq C(1+t)^{-\frac{n}{2 a}\left(1-\frac{1}{q}\right)-\frac{s}{2 a}} .
\end{aligned}
$$

Considering the singular part, we infer

$$
\left\|F_{l} * u_{0}\right\|_{L^{q}\left(R^{n}\right)} \leq C e^{-\frac{t}{2 a k}}\left\|u_{0}\right\|_{L^{q\left(R^{n}\right)}} \leq C \varepsilon e^{-\frac{t}{2 a k}}
$$

and

$$
\begin{aligned}
\int_{0}^{t}\left\|\frac{F_{l}}{1+k(-\triangle)^{a}} * u^{p}\right\|_{L^{q\left(R^{n}\right)}} \mathrm{d} \tau & \leq C \int_{0}^{t} e^{-\frac{t-\tau}{2 a k}}\|u\|_{L^{p, q}\left(R^{n}\right)}^{p} \mathrm{~d} \tau \\
& \leq C \int_{0}^{t} e^{-\frac{t-\tau}{2 a k}}(1+\tau)^{-\frac{n}{2 a}\left(1-\frac{1}{p q}\right) p} \mathrm{~d} \tau \\
& \leq C(1+t)^{-\frac{n}{2 a}\left(1-\frac{1}{q}\right)-\frac{s}{2 a}}
\end{aligned}
$$

With the help of (5.9) and (5.11)-(5.13), we obtain

$$
\|u\|_{L^{q}\left(R^{n}\right)} \leq C(1+t)^{-\frac{n}{2 a}\left(1-\frac{1}{q}\right)-\frac{s}{2 a}} .
$$

\section{Acknowledgements}

Not applicable.

\section{Funding}

This work was supported by the National Natural Science Foundation of China (No.11271141) and the Chongqing Science and Technology Commission (cstc2018jcyjAX0787).

\section{Availability of data and materials}

Not applicable.

\section{Competing interests}

The authors declare that they have no competing interests.

\section{Authors' contributions}

The authors contributed equally to the writing of this paper. All authors read and approved the final manuscript.

\section{Publisher's Note}

Springer Nature remains neutral with regard to jurisdictional claims in published maps and institutional affiliations.

Received: 7 February 2020 Accepted: 28 July 2020 Published online: 12 August 2020

\section{References}

1. Evans, L.C.: Partial Differential Equations. Graduate in Math., vol. 19. Am. Math. Soc., Providence (1998)

2. Showalter, R.E., Ting, T.W.: Pseudoparabolic partial differential equations. SIAM J. Math. Anal. 1, 1-26 (1970)

3. Barenblatt, G.I., Zheltov, I.P., Kochina, I.N.: Basic concepts in the theory of seepage of homogeneous liquids in fissured rocks [strata]. J. Appl. Math. Mech. 24, 1286-1303 (1960)

4. Benjamin, T.B., Bona, J.L., Mahony, J.J.: Model equations for long waves in nonlinear dispersive systems. Philos. Trans. R. Soc. A, Math. Phys. Eng. Sci. 272, 47-78 (1972)

5. Ting, T.W.: Certain non-steady flows of second-order fluids. Arch. Ration. Mech. Anal. 14, 1-26 (1963)

6. Padron, V: Effect of aggregation on population recovery modeled by a forward-backward pseudoparabolic equation. Trans. Am. Math. Soc. 356, 2739-2756 (2004) 
7. Rao, V.R.G., Ting, T.W.: Solutions of pseudo-heat equations in the whole space. Arch. Ration. Mech. Anal. 49, 57-78 (1972)

8. Ting, T.W.: Parabolic and pseudo-parabolic partial differential equations. J. Math. Soc. Jpn. 21, 440-453 (1969)

9. Bona, J.L., Dougalis, V.A.: An initial- and boundary-value problem for a model equation for propagation of long waves. J. Math. Anal. Appl. 75, 503-522 (1980)

10. Amick, C.J., Bona, J.L., Schonbek, M.E.: Decay of solutions of some nonlinear wave equations. J. Differ. Equ. 81, 1-49 (1989)

11. Zhang, L.: Decay of solution of generalized Benjamin-Bona-Mahony-Burgers equations in n-space dimensions. Nonlinear Anal., Theory Methods Appl. 25, 1343-1369 (1995)

12. Medeiros, L.A., Miranda, M.M.: Weak solutions for a nonlinear dispersive equation. J. Math. Anal. Appl. 59, $432-441$ (1977)

13. Cao, Y., Yin, J., Wang, C.: Cauchy problems of semilinear pseudo-parabolic equations. J. Differ. Equ. 246, 4568-4590 (2009)

14. Wang, W.K., Wang, Y.T.: The well-posedness of solution to semilinear pseudo-parabolic equation. Acta Math. Appl. Sin. 35, 386-400 (2019)

15. Kilbas, A.A.A., Srivastava, H.M., Trujillo, J.J.: Theory and Applications of Fractional Differential Equations. North-Holland Mathematics Studies, vol. 204 (2006)

16. Pu, X., Guo, B., Zhang, J.: Global weak solutions to the 1-D fractional Landau-Lifshitz equation. Discrete Contin. Dyn. Syst., Ser. B 14, 199-207 (2012)

17. Guo, B., Han, Y., Xin, J.: Existence of the global smooth solution to the period boundary value problem of fractional nonlinear Schrodinger equation. Appl. Math. Comput. 204, 468-477 (2008)

18. Li, F., Rong, F.: Decay of solutions to fractal parabolic conservation laws with large initial data. Commun. Pure Appl. Anal. 12, 973-984 (2012)

19. Wang, W.K., Yang, T.: The pointwise estimates of solutions for Euler equations with damping in multi-dimensions. J. Differ. Equ. 173, 410-450 (2001)

20. Wang, W.K.: Nonlinear evolution systems and Green's function. Acta Math. Sci. 30, 2051-2063 (2010)

21. Stein, E.M.: Singular Integrals and Differentiability Properties of Functions. Princeton University Press, Princeton (1970)

\section{Submit your manuscript to a SpringerOpen ${ }^{\circ}$ journal and benefit from:}

- Convenient online submission

- Rigorous peer review

- Open access: articles freely available online

- High visibility within the field

- Retaining the copyright to your article

Submit your next manuscript at $\gg$ springeropen.com 